\title{
Perspective
}

PERSPECTIVE Actualité en histoire de l'art

1 | 2018

Actualité en histoire de l'art

\section{La dissolution de la généalogie : Degas et Lepic, place de la Concorde}

The Mirage of Genealogy: Degas and Lepic at the Place de la Concorde Auflösung der Genealogie: Degas und Lepic am Place de la Concorde La dissoluzione della genealogia: Degas e Lepic, place de la Concorde La disolución de la genealogía: Degas y Lepic plaza de la Concordia

\section{Todd Porterfield}

Traducteur : Françoise Jaouën

\section{(2) OpenEdition}

\section{Journals}

\section{Édition électronique}

URL : http://journals.openedition.org/perspective/9551

DOI : 10.4000/perspective.9551

ISSN : 2269-7721

Éditeur

Institut national d'histoire de l'art

\section{Édition imprimée}

Date de publication : 30 juin 2018

Pagination : 145-157

ISBN : 978-2-917902-46-2

ISSN : $1777-7852$

Référence électronique

Todd Porterfield, «La dissolution de la généalogie : Degas et Lepic, place de la Concorde », Perspective [En ligne], 1 | 2018, mis en ligne le 31 décembre 2018, consulté le 01 octobre 2020. URL : http:// journals.openedition.org/perspective/9551; DOI : https://doi.org/10.4000/perspective.9551 


\section{LECTURES}

\section{La dissolution de la}

\section{généalogie: Degas et Lepic, place de la Concorde}

\section{Todd Porterfield}

Cet essai s'inscrit à contre-courant, si l'on considère le nombre croissant d'études sur l'identité et la politique identitaire ; l'intérêt que l'on porte à la question raciale chez Degas ; l'antisémitisme de l'artiste mis en lumière par Linda Nochlin dans une étude d'une magistrale précision ; les débats sur la misogynie qui ont commencé à bouillonner du vivant de Degas ; les doutes émis par une avant-garde rétrograde qui estimait que l'artiste, qui a participé à huit expositions impressionnistes et contribué à les organiser, n'était pourtant pas véritablement un impressionniste ; et enfin la riche historiographie de l'un de ses tableaux les plus célèbres, qui a fait l'objet d'âpres débats ${ }^{1}$. À travers une analyse détaillée de la Place de la Concorde (Le vicomte Lepic et ses filles traversant la place de la Concorde ; fig. 1) il s'agit de montrer que Degas a recours à des marqueurs d'identité à caractère fixe et essentiel, des généalogies, pourrait-on dire, portant sur la famille, le patriarcat, le type, le pedigree, la race, la lignée et la filiation artistique. Selon Jacques Derrida, pareille "schématisation de la filiation " constitue le concept même du politique $^{2}$. La toile de Degas les évoque tous, et on verra que, de manière surprenante peut-être pour cet artiste, ils y sont tous révélés comme parfaitement frauduleux, inefficaces et, en tout état de cause, nuls et non avenus.

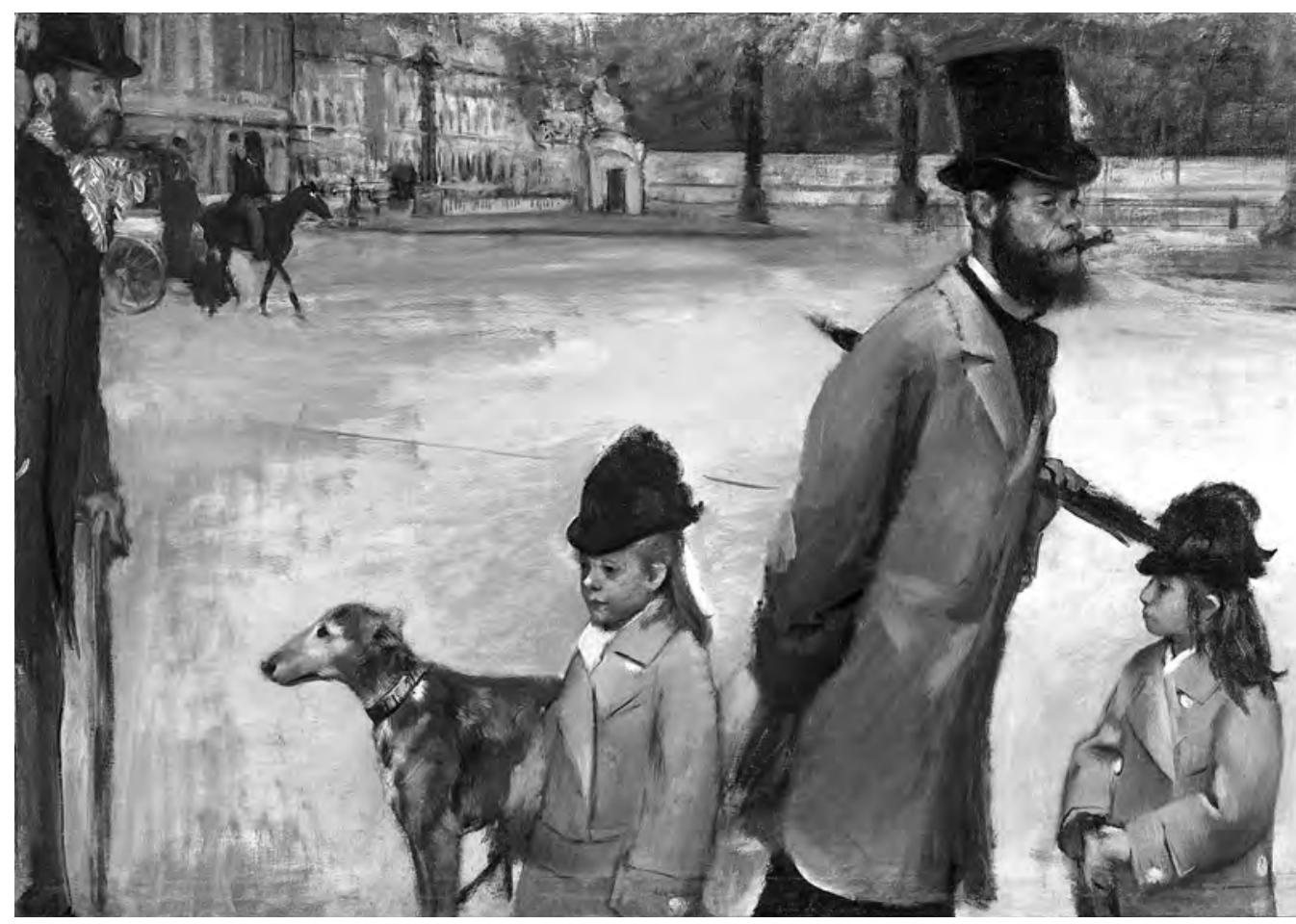

1. Degas, Place de la Concorde (Le vicomte

Lepic et ses filles traversant la place de

la Concorde), 1875, Saint-

Petersbourg, musée de l'Ermitage. 


\section{Un terrain contesté : historiographie}

Depuis près de cent cinquante ans, les analyses de la Place de la Concorde reposent sur des valeurs esthétiques ou politiques, voire civilisationnelles. Le tableau, commencé au milieu des années 1870 (et que Degas, semble-t-il, n'a jamais exposé à Paris) fut vendu en 1911 au Berlinois Otto Gerstenberg. Après la Seconde Guerre mondiale, l'armée soviétique le transporta dans les réserves de l'Ermitage, où il demeura pendant près de cinquante ans. Dans l'après-guerre, des générations d'historiens de l'art crurent que la toile avait été détruite, ne la connaissant que par des photographies, ce qui ne faisait qu'accroître l'aura de l'œuvre, considérée comme l'une des peintures les plus novatrices de son temps. Après la chute de l'Union soviétique, elle réapparut au grand jour ${ }^{3}$.

Du vivant de l'artiste, les critiques saluèrent la modernité de la Place de la Concorde. Le peintre Max Liebermann, ami de Gerstenberg et auteur d'une monographie sur Degas publiée en 1922, goûtait son naturalisme caustique et le dédain qu'elle manifestait pour la tradition académique. Aujourd'hui encore, on apprécie le caractère irrésolu de sa narration, ses formes fragmentées et l'effet d'aplatissement de l'espace, comme si (c'est ce que pensent certains), le caractère de la toile était attribuable aux effets de la photographie, l'artiste devenant ainsi à son insu un vecteur de progrès ${ }^{4}$.

Les historiens de l'art s'intéressant au social et au politique s'insurgèrent contre l'hermétisme d'un formalisme zélé, et le tableau " perdu » de Degas devint un objet fétiche pour la New Art History, tout comme pour ses adversaires. Dans les années 1980, Timothy J. Clark affirmait que la toile prenait acte de l'absence de l'histoire, reflétant la vitesse, la fragmentation et l'anomie du capitalisme ; quant à Robert Herbert, il estimait que Lepic y devenait une " distillation du flâneur » se promenant à travers une ville en plein bouleversement économique 5 . Là encore, selon ces analyses, Degas se contente dans sa toile d'enregistrer passivement des événements extérieurs.

Kirk Varnedoe a ouvert une nouvelle et fructueuse voie d'investigation qui permet de voir dans l'œuvre, non pas un accident passif, mais une démarche délibérée, en dépit de son aspect désinvolte et de l'impression de nonchalance qui s'en dégage. Évoquant le cliché selon lequel la toile impressionniste a subi l' "influence " de la photographie, Varnedoe souligne que celle-ci était à l'époque incapable de produire ce type d'effet, quoi qu'en pensent certains commentateurs, attachés à l'explication historique fournie par le déterminisme technologique ${ }^{6}$. Varnedoe, très finement, met aussi en évidence le clin d'œil de Degas, qui a dissimulé derrière le haut de forme de son ami Lepic la statue de la ville de Strasbourg, récemment annexée par les Prussiens. Celle-ci se trouve ainsi effacée de la toile tout comme elle a disparu de la carte de France. Varnedoe élargit ainsi le champ de l'analyse, qui s'ouvre à des lectures plus directes et plus politiques de l'œuvre. Dans l'espace restreint qui enferme les personnages, Albert Boime voit la trace du souvenir douloureux de la Commune ${ }^{7}$. Pour Hollis Clayson, l'œuvre s'inscrit dans le sillage du siège de Paris par les Prussiens, et les fissures de la composition traduisent la nostalgie de l'artiste pour l'esprit de camaraderie qui l'unissait à Lepic et Ludovic Halévy pendant la guerre (c'est peut-être ce dernier qui est représenté à gauche de la toile). La Place de la Concorde serait ainsi le témoignage d'un « désespoir masculin d'après-guerre " ressenti par Degas et la société dans son ensemble ${ }^{8}$. Plus récemment, André Dombrovski est parvenu à trouver un robuste équilibre entre évaluation esthétique moderniste et spécificité sociopolitique de la III ${ }^{\mathrm{e}}$ République, affirmant que l'art et la politique sont indissociables, dans une analyse partant en grande partie du chapeau de Lepic ${ }^{9}$.

Contre cette histoire de l'art sociale et politique, une guerre était déclarée. En 1985, au beau milieu des années Reagan-Thatcher, Denys Sutton publia dans la revue Apollo un éditorial intitulé "Leftspeak at Harvard ", illustré par le tableau de Degas ; il opposait le camp du connoisseurship ( "les hommes de l'estampe et du dessin »), descendant des héros Berenson et Friedländer, qui appliquaient leur érudition aux questions de style et d'attribution, à celui de Clark, professeur à Harvard, et à ses semblables qui, corrompus par la lecture de Meyer Shapiro et du Marxist Quarterly, pratiquaient l'interprétation à outrance à partir de considérations extra-artistiques. Le livre de Clark, The Painting of Modern Life, ne démontrait qu'une chose, selon Sutton, à savoir les dangers présentés par le « leftspeak" pour l'histoire de l'art ${ }^{10}$.

Sortie des sous-sols pour être accrochée dans les salles du musée à partir de 1992, la toile de Degas figurait dans l'exposition organisée en 1995 montrant les " trésors cachés " de l'Ermitage, et dont le catalogue appelait à un retour à l'ordre post idéologique, post politique et post nationaliste. Il appelait haut et fort à l'harmonie universelle afin 
d'aplanir les différends passés, prônant le rassemblement autour de valeurs artistiques (européennes) et civilisationnelles ostensiblement plus nobles. Les commissaires russes célébraient la redécouverte du tableau, véritable relique qui avait survécu à la Guerre froide, objet culturel transcendant capable de combler le fossé entre les anciens blocs de l'Est et de l'Ouest. "Pour ceux que les valeurs de la culture européenne ne laissent pas indifférents, qui voient dans la réconciliation des nations une forme de salut - pour ceux-là l'occasion de voir enfin ces tableaux si longtemps emprisonnés est un événement particulièrement émouvant ", déclarait Albert Kostenevich ${ }^{11}$. S'inscrivant dans le droit fil de ce point de vue, Mari Kàlman Meller propose en 2014 une analyse résolument stylistique de l'œuvre, récusant doublement, et de manière assez improbable, sa teneur politique. Concernant le chapeau de Lepic qui dissimule le monument de Strasbourg et la perte de l'Alsace, elle parle de simple coïncidence sans signification particulière. Pour le démontrer, elle trace un schéma en expliquant que Degas se tenant là, il ne pouvait donc voir le monument de Strasbourg. Elle se raccroche ainsi au vieux cliché selon lequel la scène impressionniste présente non seulement l'apparence de l'accidentel, mais qu'elle est entièrement le résultat du hasard ! Puis elle fournit en note une étonnante citation de Degas, qui raconte que son grand-père refusait de traverser la place de la Concorde parce que sa fiancée y avait été guillotinée sous la Révolution. Après avoir offert au lecteur cette savoureuse anecdote, elle met en garde : « Toute conséquence historique tirée de cette configuration serait, je crois, mal avisée $^{12}$. " Cette phrase fait écho à la déclaration pleine d'espoir de Louis-Philippe, qui avait décidé d'installer l'obélisque sur la place parce que, disaitil, « il ne rappellera aucun événement politique ». Comme ce dernier, Kàlman Meller soulève un point politiquement signifiant avant d'invalider toute interprétation politique. Ce n'est pas la voie que j'ai choisie.

\section{Dissolution symbolique, urbaine, picturale et familiale}

La place de la Concorde représentait l'épicentre dans l'ordre politique et symbolique français ; c'est là que les traditions artistiques avaient démontré leur incapacité à remplir leur vieille fonction, à savoir incarner, garantir et perpétuer l'ordre et la stabilité13. Le caractère éphémère des Lumières et des monuments révolutionnaires, ainsi que la succession de projets avortés - érigés puis abattus, ou construits et jamais installés marquèrent la crise de l'art à la période moderne. $\mathrm{Ni}$ la "place royale » construite sous l'Ancien Régime, réaménagée jusqu’à la Seine par Gabriel et rendue accessible au trafic commercial, ni la statue équestre du roi remaniée dans un style plus naturaliste par Bouchardon et dotée d'une iconographie pacifique, ne purent prévenir l'iconoclasme et le régicide révolutionnaires. Puis les gouvernements et les monuments se succédèrent. Après la Liberté de plâtre et la machine guillotine de la Révolution (fig. 2), Napoléon, prudent, voyait d'un œil méfiant les commémorations permanentes. La Restauration tenta ensuite une nouvelle statue de Louis XV, suivie d'une réincarnation de Louis XVI montant au ciel. Louis-Philippe choisit une solution lourde de sens en optant pour un monument bien plus ancien, l'obélisque pharaonique, manière d'éviter les conflits épineux relatifs aux récits nationaux et aux monuments de tous types en leur substituant une célébration très moderne des conquêtes impériales invoquant le discours de la supériorité civilisationnelle qui serait, au cours des décennies suivantes, renforcé par le discours du racisme, ce à quoi la toile de Degas renonce.

Le tableau distille un malaise étroitement lié à l'histoire de la place. Durant des décennies, ce trouble donna lieu, dans ses représentations, à des jeux formels exagérant à outrance ou au contraire diminuant l'échelle de la place et de son mobilier urbain, comme si l'on pouvait, par ces exercices d'imagination, maîtriser la peur et les souvenirs
2. Pierre-Antoine Demachy (attr.), Une exécution capitale, Place de la Révolution, 1793-1794, Paris, musée Carnavalet. 
3. William Wyld, La Place de la Concorde, vers 1838 , aquarelle avec rehauts de gouache, Paris, musée Carnavalet.

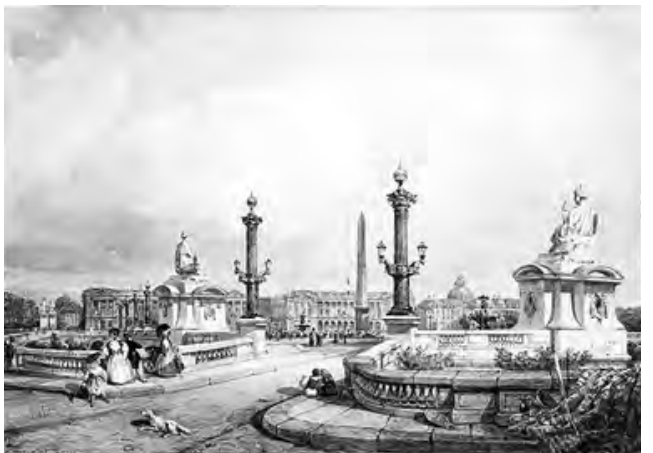

de violence attachés au site. Sous la monarchie de Juillet, une caricature soulignait le vide en grossissant le monolithe géant, qui devenait une quille colossale de bowling. En 1844, une comtesse espagnole qui se lamentait à propos de l'ascension de la bourgeoisie en France compara le décor de la place de la Concorde (qu'elle continuait à appeler place Louis XV), avec son obélisque, ses fontaines, ses lampadaires et ses statues, à un gigantesque surtout de table ${ }^{14}$. Le tableau de Degas ne montre pas une image plus rassurante de la place que ces représentations. Il ne confère à Lepic aucun ancrage, le laissant à la dérive sur un flot de sensations, sur la place d'une concorde quasi inexistante.

L'angoisse provoquée par le vide de la place ne fait qu'empirer avec les modifications apportées en 1854 par Napoléon III. Vers 1838, William Wyld peint une aquarelle qui accentue l'aspect relativement plaisant du cadre avant haussmannisation (fig. 3). Les lampadaires à colonnes rostrales polychromes donnent un rythme à la statuaire et au monolithe, offrant des points d'accroche dans la profondeur et à la surface du champ pictural urbain, tandis que les fossés plantés estompent la minéralité de la surface et de l'élévation et que les formes courbes des balustrades arrondissent les arêtes de l'implacable géométrie du grand axe, adoucie par un tapis oriental posé en travers d'un rebord comme un jeté de divan dans un boudoir. Au début du Second Empire, Hittorff propose de renforcer le lyrisme pastoral en ajoutant des balustrades, des pelouses, des dénivelés et des vases de bronze, mais l'empereur s'y oppose, et ordonne à Haussmann de combler les fossés et de supprimer les balustrades afin de faciliter la circulation ${ }^{15}$.

En 1854, L'Illustration se lamente à propos de la place, devenue le centre du chic parisien après les travaux de Gabriel :
C'est aujourd'hui une sorte de voyage aventureux, que d'aller d'un trottoir à un autre, et de traverser la place pour se rendre aux Tuileries et aux ChampsÉlysées ou dans telle autre direction. Au milieu d'un mouvement rapide et croisé de nombreux équipages se précipitant dans toute la largeur de la nouvelle voie ouverte, il est déjà assez difficile pour un homme de s'y diriger en évitant les obstacles, et cela exige une attention inquiète de tous les instants; mais cela devient une chose dangereuse pour une femme, surtout si elle a des enfants ${ }^{16}$.

La nouvelle configuration de l'espace au Second Empire écrase ainsi le flâneur et efface les lignes qui auraient pu fournir un cadre rassurant à sa promenade ${ }^{17}$. La toile de Degas accentue cet effet nouveau d'anxiété. Projeté contre un paysage urbain qui menace de l'annihiler, le groupe familial est marginalisé et la plus grande partie de l'espace est vide. Derrière lui, l'espace se dresse, vertigineux et, autour de lui, tournoie une parabole dont le mouvement s'amorce dans le coin inférieur gauche et menace de l'engloutir. Le tableau du cœur monumental de Paris est dominé par l'absence et le vide.

Il est étrange que Degas - ou tout autre impressionniste français - ait choisi de peindre une place publique chargée d'histoire, même si les artistes italiens vivant à Paris (De Nittis, Signorini et Boldini) s'en étaient fait une sorte de spécialité au début des années $1870^{18}$. Degas, qui s'intéressait de près à leur travail, les poussa à exposer aux salons impressionnistes ${ }^{19}$. Après le siège de Paris et les massacres de la Commune, ils célébraient la vitalité retrouvée de la ville et de la nation tout entière.

Giuseppe De Nittis, notamment, peignit quantité d'images des places parisiennes, et Degas conçut probablement son tableau en dialogue avec deux de ses toiles, peintes en 1875 : une Place des Pyramides (fig. 4) d'assez belle allure et une Place de la Concorde un peu moins intéressante ${ }^{20}$. La première représente une tranche de vie urbaine affairée montrant des bourgeois en promenade, des blanchisseuses livrant leur linge, une charrette d'oranges, des panneaux publicitaires et un omnibus qui passe, le tout assemblé dans une agitation collective et cohérente. On y découvre le résultat de la destruction du palais des Tuileries, mais l'échafaudage accroché au pavillon du Louvre semble indiquer que les Parisiens sont soucieux de reconstruire le cœur monumental de leur ville en signe de retour à la normale, inspirés par la 
figure énergique de Jeanne d'Arc. On note également - et ce n'est pas un hasard - la présence des Lepic, qui prennent part, elles aussi, à cet hymne à la guérison nationale : au premier plan, les deux filles, accompagnées d'une femme vêtue à la dernière mode (sans doute leur mère), sont associées au charmant détail d'un petit griffon qui file sur les pavés glissants. Cette image de la famille Lepic menée par un seul parent trouve sa répartie dans la toile de Degas.

Les Lepic, mariés en 1865, commencèrent à vivre séparément dès le début des années 1870. En 1867, Ludovic prit une maîtresse, qui l'accompagnait dans sa vie mondaine. Au début des années 1870, on discutait âprement d'une éventuelle légalisation du divorce ; il suscitait l'opposition de ceux qui voyaient dans la Commune un désastre ayant entraîné le déclin de l'autorité familiale et une rébellion des masses ${ }^{21}$. Après la naissance de la troisième fille Lepic en mars 1874, le couple se sépara définitivement, une séparation qui devient officielle en 1881. Ils

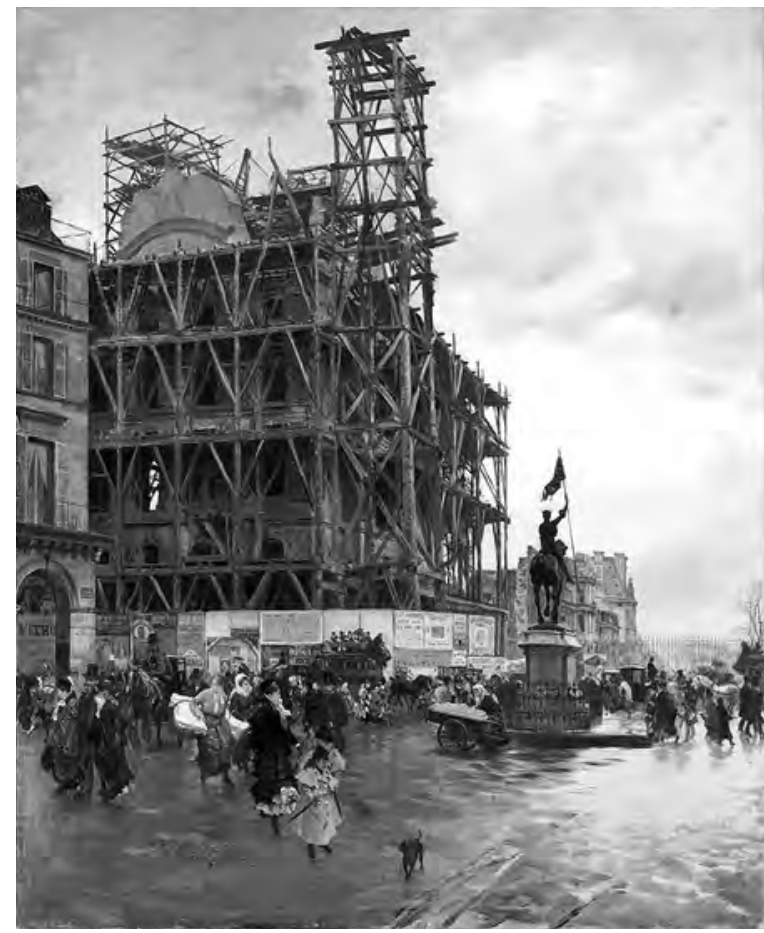

4. Giuseppe De Nittis, Place des Pyramides, 1875, Paris, musée d'Orsay. divorcent en $1885^{22}$, quelques mois à peine après le vote de la loi Naquet (1884) légalisant le divorce en France pour la première fois depuis Napoléon I ${ }^{\text {er23 }}$. Degas, qui a représenté plusieurs fois son ami Lepic en compagnie de ses filles, mais jamais de sa femme, ne semble pas déplorer l'érosion de l'autorité patriarcale et ne fait rien pour la restaurer. Entre père et filles sur la place de la Concorde, on note des fissures et des vides. Ludovic paraît désinvolte et distrait, contrairement à Eylau et Jeanine, plus attentives et posées. Eylau, serrée dans son manteau croisé, se comporte comme le soldat que son père a cessé d'être.

Degas aurait pu, en choisissant la place de la Concorde, peindre un sujet auquel s'attachaient des souvenirs, des emblèmes et des trophées personnels, nationaux ou impériaux. Le monument de la ville de Strasbourg, prise par les Allemands en 1871, est devenu un site de pèlerinage couvert de couronnes, de bannières et d'inscriptions ${ }^{24}$. L'artiste aurait pu remplir sa toile d'emblèmes du classicisme architectural et artistique français, mais il choisit de ne montrer qu'un fragment isolé du palais de la Marine. Il réduit à d'étroits bandeaux les murs et les balustrades du jardin des Tuileries. Même l'ornement principal de la place, l'Obélisque de Louxor, est absent. Il ne reste que la statue de Lille, assise sur un piédestal creux.

\section{Le pedigree et la race}

Parmi toutes ces absences, Degas fournit une série de marqueurs de filiation. Le vicomte LudovicNapoléon Lepic lui-même incarne éminemment la notion de pedigree, selon un article publié en 1877 dans La Galerie contemporaine. Le profil de personnalité brossé par le magazine introduit Lepic, en employant une rhétorique généalogique et masculine, comme l'aide-de-camp de Napoléon III, fait prisonnier par les Prussiens pendant la guerre, issu d'une "grande race de soldats $^{25}$ ". Le grand-père, héroïque à la bataille d'Eylau, avait été fait baron et décoré de la Légion d'honneur, et son nom avait été inscrit sur l'Arc de Triomphe ${ }^{26}$. Le père de Ludovic, député à l'Assemblée nationale, avait soutenu le coup d'État de Napoléon III, avant d'être nommé premier maréchal des logis et surintendant des palais impériaux, il avait donc supervisé la décoration des Tuileries. En 1875, le palais, incendié pendant la Commune, constitue une absence significative, à la fois dans la ville et dans le tableau. Il était situé au-delà du mur qui ferme la perspective dans la toile de Degas. Dans cette configuration lourdement bonapartiste, Ludovic et ses filles - la plus jeune, Jeanine, et l'aînée, qui porte un trio de 
5. Léon Crémière 4e Prix, Médaille de bronze, Abreckt, mai 1863 , photographie positive sur papier albuminé d'après un négatif sur verre au collodion, dans Jardin zoologique d'acclimatation (Bois de Boulogne). Exposition de Chiens. Mai 1863 (portfolio de 60 planches), Paris, BnF, département des Estampes et de la Photographie, PET FOL-KE-30 (B).

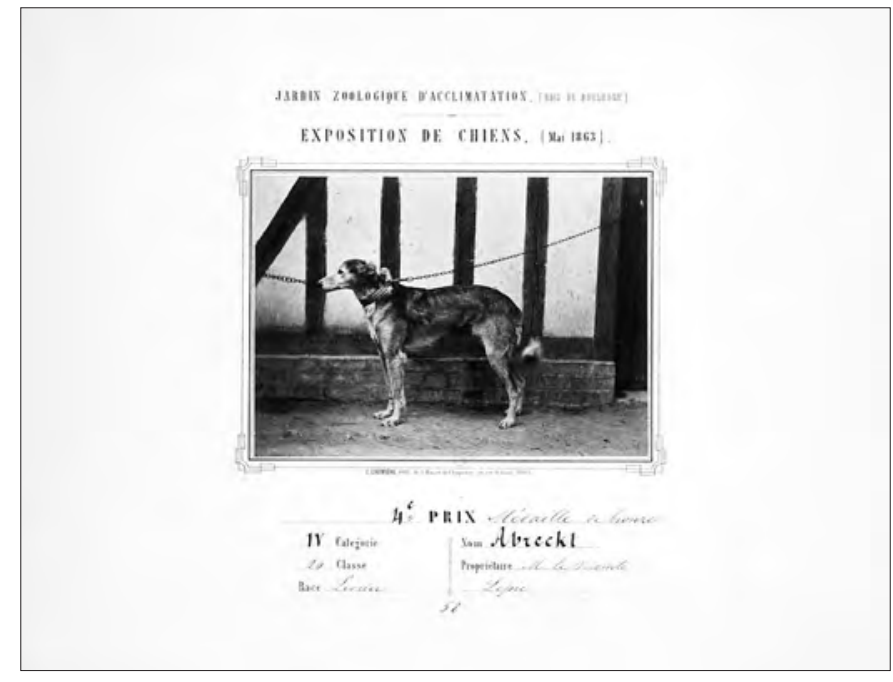

et en Angleterre se tourne de plus en plus vers la quête d'une essence immuable, d'un modèle naturel et incontestable pour une " société stable et hiératique où le rang social est assuré31 ". Peu à peu, la dimension culturelle des recherches sur les races canines prend une autre tournure, comme en témoignent les textes et les illustrations des catalogues des expositions canines organisées entre les années 1860 et les années $1880^{32}$. Ils témoignent d'un évident glissement stylistique par rapport au passé, où l'on préférait un naturalisme rural pittoresque, où les chiens étaient représentés

noms napoléoniens (Eylau-Eugène-Hortense) tournent le dos aux ruines distantes du palais qui fut leur foyer jusqu'en $1870^{27}$.

Durant ses loisirs, Lepic adorait se plonger dans l'étude des pedigrees. En 1863, il fut l'un des mécènes de la première Exposition universelle canine de Paris, où son lévrier Abreckt remporta une médaille, ce qui valut à l'animal un portrait réalisé par Léon Crémière, photographe officiel de la maison impériale ${ }^{28}$ (fig. 5). Ce portrait trouve un écho dans le soigneux profil du lévrier de la Place de la Concorde, qui arbore un collier strié similaire, et dont les yeux et les oreilles sont de même proportion (mais dont le museau est moins prononcé). Dans la photographie officielle de Crémière, des chaînes aident le chien médaillé à garder la pose, tout comme dans l'atelier d'un peintre académique, des chaînes et une poulie aident le modèle à se tenir immobile. Dans le tableau de Degas, ces chaînes ont disparu, mais le statisme de la pose pleine de noblesse a été conservé.

Le chien du tableau est un emblème de la quête de pureté et d'antiquité qui constitue la base idéologique de la notion de pedigree. En 1863, Albert Geoffroy Saint-Hilaire, président de l'Exposition universelle canine et directeur du zoo impérial, annonce que l'exposition a pour but d'établir des distinctions entre les races et d'identifier les races croisées dignes d'être préservées ${ }^{29}$. À la même période, le Britannique Francis Galton utilise l'élevage de chiens pour mettre au point ses théories eugénistes concernant l'homme ${ }^{30}$. Après la guerre de 1870-1871 et la Commune, l'intérêt des classes aisées pour les races animales en France de trois quarts dans un cadre naturel. Au fil des décennies, les illustrations privilégient des profils de plus en plus précis, adoptant la rhétorique visuelle du spécimen scientifique.

\section{Iconographie désuète}

On peut se demander si le tableau de Degas et les lévriers médaillés de Lepic ne contribuent pas à affirmer une hiérarchie sociale justifiée par la race. Dans l'univers de l'élevage canin, le lévrier est considéré comme l'une des races " dont l'antiquité est la plus grande ${ }^{33}$ ". À Paris, le "véritable quartier nobiliaire de la race canine" a pour centre la place de la Concorde, où l'aristocratie elle-même peut être qualifiée de «tribu de lévriers ${ }^{34}$ ". En histoire de l'art, le portrait canin, comme le portrait équestre, traduit depuis longtemps la noblesse du maître, comme on peut le voir dans le tableau de Courbet, Les Lévriers du comte de Choiseul (1866), où les chiens remplacent le maître absent ${ }^{35}$. Dans le tableau de Degas, en revanche, le pedigree pose problème, et d'autres précédents n'ont rien de rassurant.

Déjà sous la Révolution, la suprématie illégitime et précaire de la noblesse avait fait, en 1793, l'objet d'un pamphlet intitulé Lettre d'un chien aristocrate à son maître, dans lequel le chien montrait à l'homme lequel des deux avait la condition la plus assurée. Au maître qui, terrifié par les émeutiers, s'était enfui de sa demeure ancestrale, le chien disait : "Apprenez que ma race, que ma noblesse sont aussi anciennes, aussi pures que race et noblesse d'Europe, qu'elles reposent sur les mêmes bases : l'impudence d'une part, la crédulité 
de l'autre ${ }^{36}$. " Le texte et ses illustrations traçaient une généalogie qui dotait le chien d'un pedigree plus ancien et plus solide que celui de l'aristocrate, puisqu'il remontait à la Louve romaine du Capitole.

Dans le tableau de Degas, le lien entre chien et maître n'est ni assuré ni rassurant. La composition de groupe ne ressemble en rien, par exemple, à la statue du prince impérial réalisée par Carpeaux en 1866-1867, une œuvre où le chien, Néron, s'enroule autour du jeune prince, apportant soutien et crédibilité à l'héritier de cette douteuse dynastie $^{37}$ (fig. 6). Ici, au contraire, la noblesse du lévrier est formellement dissociée du vicomte Lepic, personnage à pedigree, qui est fâcheusement écarté.

Avec ironie, Degas et Lepic jouaient tous deux sur le fossé existant entre chien et maître. Le premier avait ainsi adressé une lettre à « M. Lepic, fournisseur de bons chiens ${ }^{38}$ " pour lui demander un petit chien de compagnie destiné à Mary Cassatt. Quant à Lepic, ses premières incursions publiques sur la scène artistique étaient des variations spirituelles sur la seule noblesse qui vaille, celle du pedigree artistique et canin. Il fit ses débuts au Salon de 1861, où il remporta un prix avec ses eaux fortes copiées d'après les " chiens héroïques " de Louis-Godefroy Jadin, peintre de la Vènerie impériale, surnommé « le Michel-Ange des chiens ${ }^{39}$ ". Les gravures de Lepic, comme par exemple le César de 1861, témoignent de manière drolatique de l'épuisement de la rhétorique classique, et de l'importance infinitésimale du pedigree artistique.

\section{La généalogie artistique}

Le chien de Lepic nous arrive aussi doté d'un pedigree qui renvoie à la Renaissance italienne, que personne ne semble avoir remarqué jusqu'ici. $\mathrm{Au}$ pedigree et aux affiliations familiales ou raciales, il faut donc ajouter à la filiation artistique du lévrier un détail d'une fresque réalisée à Vérone par Pisanello, Saint Georges délivrant la princesse de Trébizonde (1436-1438). Degas n'a peut-être jamais vu cette peinture, en dépit de multiples voyages en Italie à partir de 1856. Il rend visite à sa famille à Naples et se rend à Rome, Florence, Pise, Sienne, Turin et dans d'autres capitales artistiques en l'espace de trois années. Il y fait de nouveaux séjours en 1860, 1875 et 1876, mais on ignore s'il s'est rendu à Vérone ${ }^{40}$. Il est en revanche un habitué du Louvre, où il réalise six cents copies de maîtres, parmi lesquelles des copies d'après le Codex Vallardi, "splendide

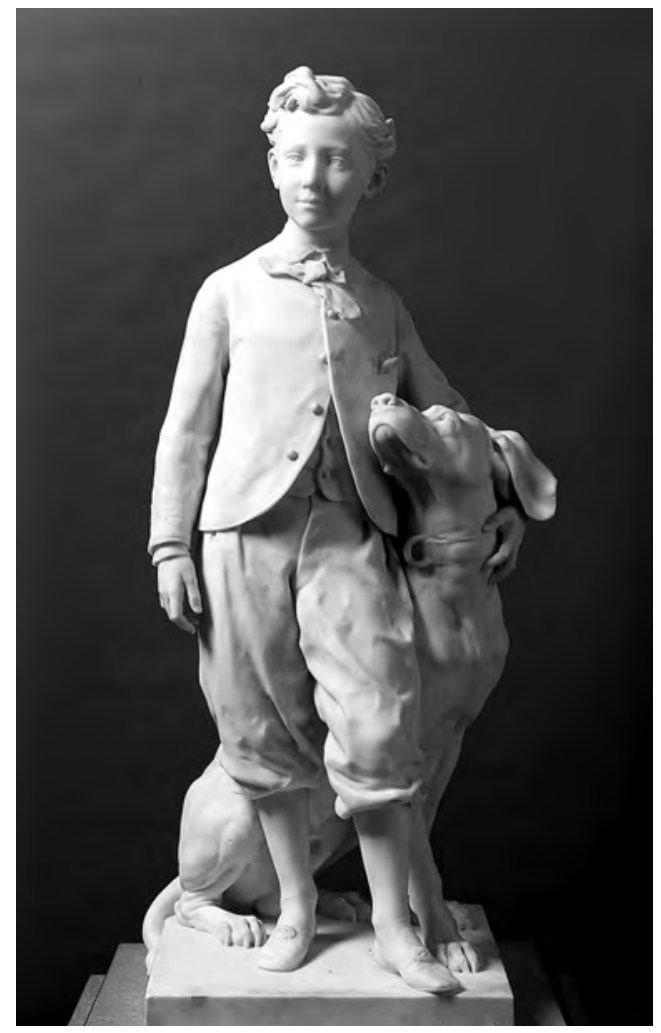

6. Jean-Baptiste Carpeaux, Le Prince impérial et son chien Néro, 1865, Paris, musée d'Orsay.

apogée de l'histoire du dessin de livres de modèles italiens ${ }^{41}$ ". Acquis par le Louvre en 1856, il est alors attribué à Léonard de Vinci, avant d'être réattribué à Pisanello et à son atelier, deux ans après la réalisation de la toile de Degas ${ }^{42}$. Le Codex contient la quasi-totalité des dessins de Pisanello, parmi lesquels cinq dessins de lévriers, dont deux ressemblent étroitement à ceux de la fresque de Vérone et de la toile de Degas (fig. 7).

Le tableau de Degas est marqué par la figure du chien de Pisanello, modèle d'immobilité, de fermeté et de raideur. Même si son lévrier diffère par plusieurs détails de ceux du Codex représentés de profil, et qu'il se rapproche davantage de l'animal de la fresque de Vérone et de la photographie de Crémière, il partage avec ceux du Codex la noblesse et la fermeté de la posture de l'animal et du spécimen artistique. Contrairement à d'autres passages du tableau - exécution rapide, postures contingentes, mode vestimentaire éphémère, personnalité contemporaine, figures apparaissant dans le champ de vision et disparaissant tout aussi vite - le lévrier de Lepic est le seul élément de la composition présenté de profil et il est la seule créature qui ne soit pas en mouvement. 


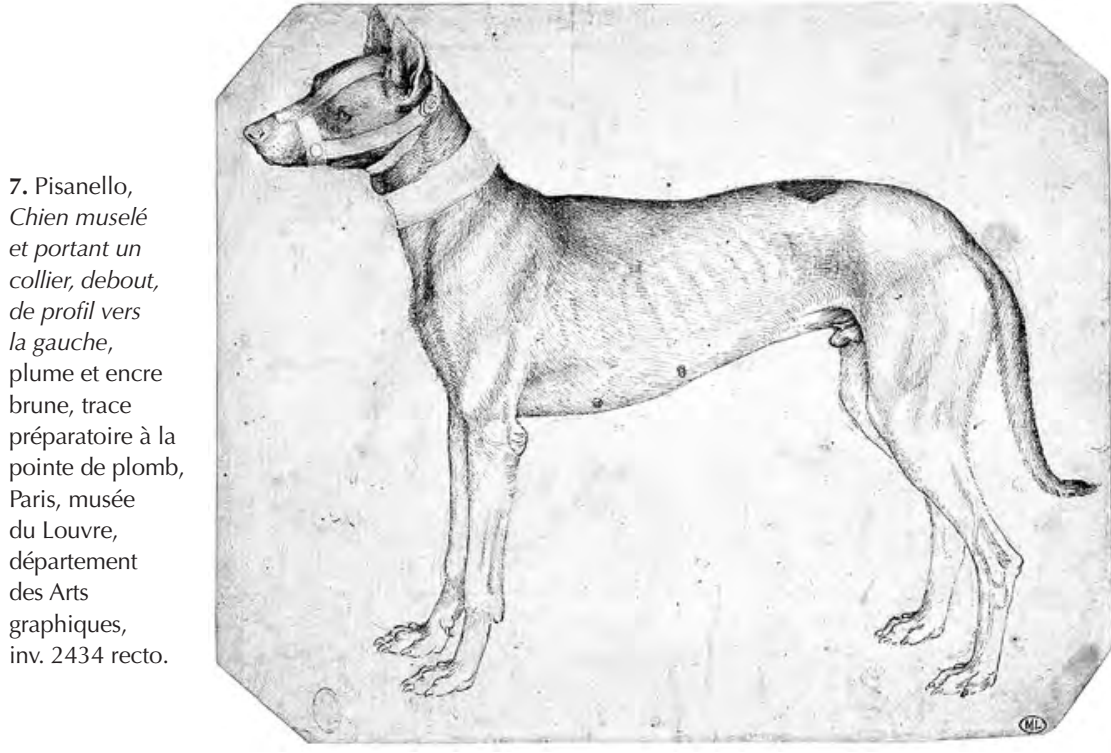

Contrairement aux trois Lepic - Ludovic et ses filles, représentés de trois-quarts et tournés dans des directions différentes - le chien semble ancré au sol. Son immobilité et la rigidité de sa posture ont quelque chose de la figure égyptienne. La pose de sa tête établit une ligne de vision qui épouse rigoureusement la surface du tableau. L'animal est le seul à se soumettre au plan de la toile.

À la fin des années 1850, à l'époque où Degas copiait le Codex Vallardi, l'artiste et son père partagent des intérêts communs, et on glisse ici de la généalogie artistique à la généalogie filiale. À son fils qui voyage en Italie, Auguste de Gas conseille la contemplation de " ces adorables maîtres fresquistes du $\mathrm{XV}^{\mathrm{e} 43}$ " et, un an plus tard, souligne l'importance des " maîtres du XV [qui] sont les véritables seuls guides ${ }^{44}$ ". Dans les années 1850 , les Degas sont attirés par ce que l'on considère alors comme une sorte de primitivisme, une pureté archaïque, modèle d'immobilité, encore vierge des robustes effets modernisateurs de Raphaël et de Michel-Ange.

La référence de Degas au Quattrocento par le biais de dessins issus de livres de modèles est surdéterminée, car le genre du livre de modèles est lui-même une machine à fabriquer des généalogies artistiques. On sait à quel point ces compendia sont des outils indispensables de la pratique artistique à la fin du Moyen Âge et au début de la Renaissance et jouent, avant l'ère de la reproduction mécanique, un rôle essentiel en perpétuant l'autorité, les schémas et l'exemplarité des grands modèles artistiques ${ }^{45}$. Curieusement, alors qu'ils visent à garantir la stabilité de la pratique artistique et à préserver le caractère distinct du modèle, ces manuels destinés à l'imitation recèlent aussi un potentiel générateur. Pisanello se servait du parchemin parfois translucide pour copier, décalquer et inverser des figures de chiens et de lions dans les pages consultées par Degas ${ }^{46}$. Au fil des décennies, ce dernier a de plus en plus eu recours à la multiplicité, à l'inversion, au renversement et à l'agrandissement, autant de techniques qui le conduisent très loin de toute source faisant autorité.

Mais c'est cet attachement au Quattrocento et à son anti-modernisme présumé que les partisans réactionnaires de Degas ont tout particulièrement apprécié. Dans une thèse sur le classicisme dans la peinture française (sujet suggéré par Maurice Denis) publiée en 1931, Robert Rey, conservateur à Fontainebleau, déclare ainsi à propos de Degas : " Notre race trouvera le motif de beaucoup de confiance en soi-même ${ }^{47}$ ", comme si le classicisme de l'artiste était un antidote à la dégénérescence de la modernité. Le commentaire de Rey met en évidence la machinerie discursive de l'histoire de l'art qui a longtemps servi à fabriquer des identités et des hiérarchies civilisationnelles et raciales $^{48}$. En contemplant la Place de la Concorde, on s'étonne de lire cette phrase chez Rey, qui fait de Degas un virulent anti-impressionniste : "Il voudrait qu'on fusillât les "Impressionnistes" férus de ce "plein-air", esclaves de cette nature prise sur le vif et qu'il abhorre comme étant un simple artifice photographique ", clame-t-il. Rey reconnaît que Degas a connu une phase réaliste, mais pardonne, en définitive, l'artiste, parce qu'il vivait entouré d'œuvres d'Ingres, Delacroix, Corot et Cézanne, c'est-à-dire une lignée généalogique $\mathrm{d}$ 'art certes moderne, mais national et classicisant. Mais une lecture attentive du tableau de Degas montre que celui-ci correspond assez mal à l'idée que s'en fait Rey.

En effet, la généalogie artistique présentée dans la toile reste sans effet; c'est une farce, un mirage, et Degas ne manifeste qu'ironie et indifférence devant ce constat ${ }^{49}$. Tout est arrangé 
sur la place de la Concorde, site emblématique de l'histoire de France, comme si le chien de la famille était le seul monument à conserver un dernier reste d'autorité, ce qui ne manque pas de piquant. Des siècles de tradition artistique aboutissent à un médaillé de bronze d'une exposition canine parisienne. Le vicomte Lepic et ses filles traversant la place de la Concorde est une machine qui montre l'insuffisance, l'inefficacité et l'absurdité de l'identité innée.

\section{Stabilité sociale incompatible}

Loin d'évoquer un sentiment de nationalisme transcendant, d'ordre et de hiérarchie sociale rassurants que, pendant des siècles, beaucoup ont cherché sur la place et dans le classicisme français, la Place de la Concorde est sans doute le plus radical des tableaux de Degas, précédant les splendides dessins réalisés par le jeune Georges Seurat au début des années $1880^{50}$, dessins où le vide domine. Dans la Place de la Concorde, l'hiver (fig. 8), l'une des fontaines semble flotter dans l'espace pictural. L'élément majeur de la composition est tronqué et donne l'impression étrange d'être attaché à un fiacre qui traverse la place en le tirant. Un fragile lampadaire isolé s'efforce de stabiliser la scène. À l'arrière-plan, d'autres verticales pourraient aider à cette tâche, mais elles sont trop fantomatiques et n'offrent aucun répit, ni à l'image, ni à la place, et encore moins à la nation. Elles sont tout aussi irrémédiables que le lévrier de Degas (et de Pisanello), incapables d'arrêter le mouvement, trop insignifiantes pour remplir le vide.

Les articles publiés par Georges Bataille en 1929-1930 dans Documents pointent dans la même direction ${ }^{51}$. "Les grands monuments s'élèvent comme des digues opposant la logique de la majesté et de l'autorité à tous les éléments troubles ", écritil dans une décapante critique des monuments, de l'architecture et du pedigree classique en peinture. La fonction sociale de l'architecture est réitérée dans le musée et sur la place publique, comme elle l'est dans la peinture traditionnelle, qui démontre « un goût prédominant de l'autorité humaine ou divine ". Selon Bataille, " les grandes compositions de certains peintres expriment la volonté de contraindre l'esprit à un idéal officiel ", tandis

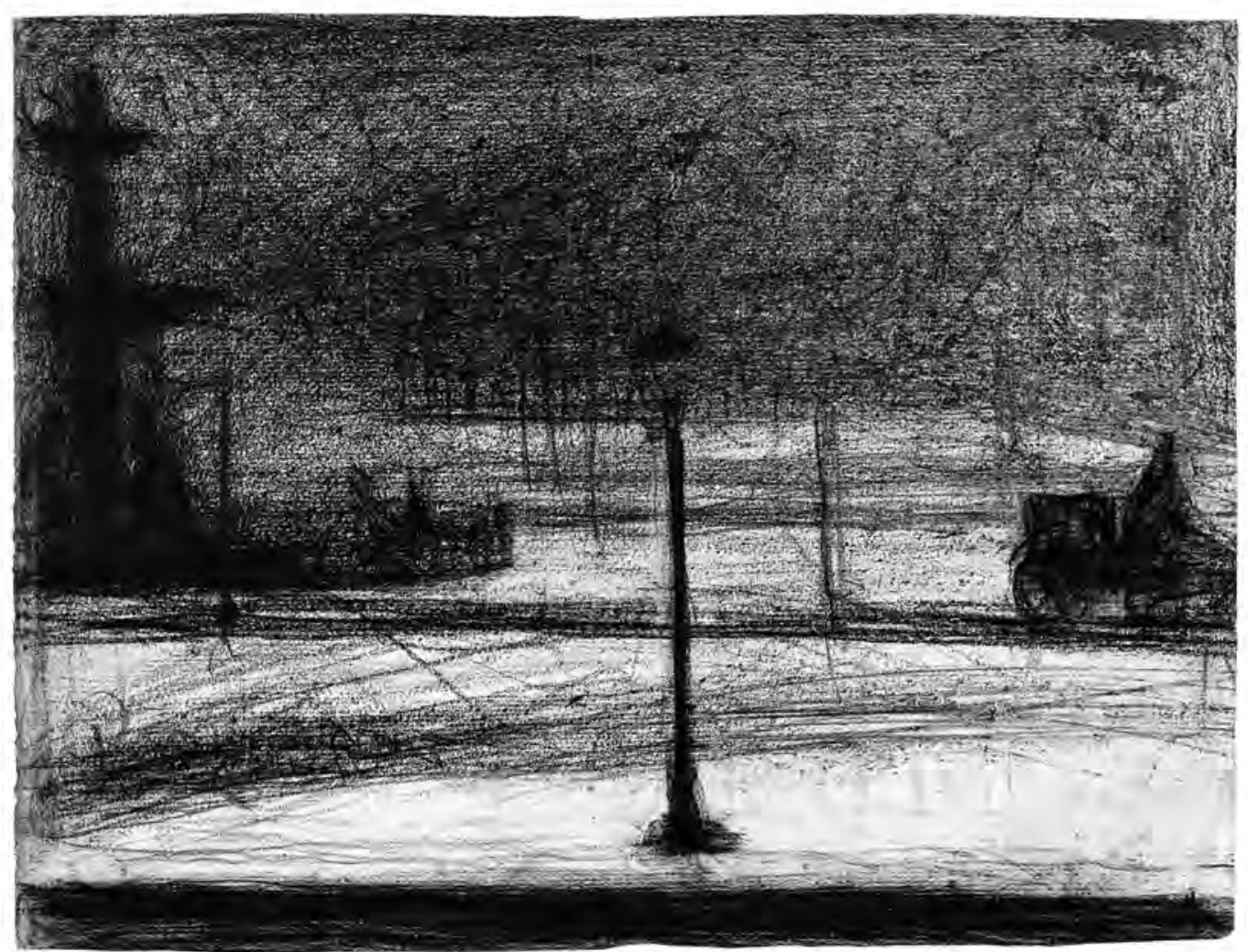

8. Georges Seurat, Place de la Concorde (hiver), 1882-1883, New York, Solomon R. Guggenheim Museum. 
que « la disparition de la construction académique en peinture est, au contraire, la voie ouverte à l'expression (par là même à l'exaltation) des processus psychologiques les plus incompatibles avec la stabilité sociale ${ }^{52} "$. La célèbre toile peinte en 1875 par Degas produit précisément cela. Elle met en scène l'inefficacité de la généalogie, qu'elle soit artistique, familiale ou raciale.

Dans l'œuvre de Degas, le classique n'est présent qu'en tant que vestige, démontrant son incapacité à préserver l'ordre social, racial et artistique. Seul le descendant d'un chien lauréat d'un concours nous rattache à la mémoire et à l'autorité des nobles races et à la généalogie artistique de la Renaissance. Tous ces pedigrees sont impuissants dans le vortex de la place que Bataille appelait l'incarnation de la perte, où la guillotine laissait entrer des bourrasques hurlantes et glacées. Certains y verraient une perte. Pour d'autres, nés à l'écart des illusions de la généalogie, de la race ou du pedigree, ou pour ceux disposés à y renoncer, il s'agirait plutôt d'une ouverture.

\section{Le texte a été traduit de l'anglais par Françoise Jaouën}

Todd Porterfield, New York University tp32@nyu.edu 
1. Dans une analyse lucide et perspicace, Linda Nochlin a mis en lumière l'antisémitisme de Degas ( Degas and the Dreyfus Affair: Portrait of the Artist as an Anti-Semite " [1987], dans The Politics of Vision: Essays on NineteenthCentury Art and Society, New York, Harper and Row, 1989, chap. VIII). Sur la question raciale, voir Marilyn R. Brown, " "Miss La La's" Teeth: Reflections on Degas and "Race" ", dans The Art Bulletin, vol. 89, n 4, décembre 2007, p. 738-765 ; James Smalls, " "Race" as Spectacle in Late Ninetenth-Century French Art and Popular Culture ", dans French Historical Studies, vol. 26, n 2, printemps 2003 , p. 351-382.

2. Jacques Derrida, Politiques de l'amitié, Paris, Galilée, 1994 cité par François Noudelmann, "Introduction ", dans Robert Harvey, E. Ann Kaplan et François Noudelmann (dir.), Politique et filiation, Paris, Kimé, 2004 ; Noudelmann ajoute : "La déconstruction du généalogique n'est cependant qu'une étape et ne peut nous dispenser de réfléchir, politiquement, à la définition du lien communautaire. Cette réflexion suppose précisément de convoquer à nouveau les notions de représentation, de filiation et d'affiliation, de modèle et de ressemblance" (ibid., p. 10).

3. Le tableau fut cédé lors de la vente Lepic le 22 mars 1897 ; Degas voulait y ajouter « quelques touches qui lui manqu[ai]ent " en 1898, mais ne l'avait toujours pas fait en octobre 1904. Durand-Ruel le vendit à Gerstenberg, en décembre 1911 (Caroline Durand-Ruel, « Lettre de Degas conservées dans les archives Durand-Ruel ", dans Degas inédit, actes du colloque Degas, actes du colloque (Paris, musée d'Orsay/École du Louvre, 1988), Paris, La Documentation française, 1989, p. 472. En 1935, Margarethe Scharf hérita de la collection de son père. En 1943, la toile de Degas ainsi que d'autres furent envoyées à la Galerie nationale de Berlin puis, à l'été 1944, transférées dans la tour de défense anti-aérienne (Flatkurm) située près du zoo de la ville. Sur sa provenance, voir la documentation fournie dans l'excellent ouvrage commémoratif dirigé par Janina Dahlmanns, Julietta Scharf et Hannah Strodza, Die historische Sammlung Otto Gerstenberg, Berlin, Hatje Cantz 2012, 2 vol. Voir également Hanna Strodza, "Verkauft - Verbrannt - Verschleppt - Verschollen, Margarethe Scharf und des Shicksal der Sammlung Gerstenberg nar 1935 ", dans ibid., vol. 1, p. 78-95, et Werner Hofmann, "Edgar Degas: Graf Lepic und Seine Töchter. Place de la Concorde ", ibid., vol. 1, p. 166-181.

4. L. Houtrticq, "E. Degas", dans Art et décoration : revue mensuelle d'art moderne, $\mathrm{n}^{\circ}$ 32, juillet-décembre 1912, p. 97113 ; Paul-André Lemoisne, Degas, Paris, Librairie centrale des beaux-arts, 1911, p. 55-56; Max Liebermann, Degas, Berlin, Bruno Cassirer, 1922, p. 19-22 (avec une reproduction). Le jugement porté sur la toile par Lieberman est en accord avec le goût de Gerstenberg, qui possédait une collection exceptionnelle d'œuvres de Daumier et de Toulouse-Lautrec. Pour Duranty, les qualités formelles du tableau " constituaient les caractéristiques fondamentales de l'art moderne " (dans La Nouvelle peinture, Paris, Floury, 1876, p. 38), comme le signale Charles F. Stuckey dans "Degas, Artiste : sans cesse corriger et jamais achever ", dans Maurice Guillaud (dir.), Degas, le modelé et l'espace, Paris, Centre Culturel du Marais, 1984, p. 13-71. À propos de l'influence présumée de la photographie sur l'impressionnisme, voir Aaron Sharf, Art and Photography, Baltimore, Penguin, 1968.

5. Timothy J. Clark, The Painting of Modern Life: Paris in the Art of Manet and his Followers, Princeton, Princeton University Press, 1984, p. 75-78 ; Robert L. Herbert, Impressionism: Art, Leisure, and Parisian Society, New Haven,
Yale University Press, 1988, p. 33-35, 50-57. Werner Hofmann reprend le thème du flâneur dans " A Painted Parade ", dans Degas: A Dialogue of Difference, David H. Wilson (Eng. transl.), Londres, Thames \& Hudson, 2007, p. 105-117; c'est également le cas de Peter Brix Søndergaard dans "Blikkets Modernisering. Edgar Degas' Vicomte Lepic, Place de la Concorde ", dans Synvinkler på kunsthistorien, 1991, p. 198-145 (merci à Jesper Rasmussen qui m'a aidé à traduire ce texte).

6. Kirk Varnedoe, "The Artifice of Candor: Photography and Impressionism Reconsidered ", dans Art in America, janvier 1980, p. 66-78; idem, "The Ideology of Time: Degas and Photography ", dans Art in America, mai-septembre 1980, p. 96-110. Françoise Heilbrun rejoint l'analyse de Varnedoe ; voir "Sur les photographies de Degas ", dans Degas inédit..., 1989, cité n. 3, p. 159.

7. Albert Boime, Art and the French Commune: Imagining Paris after War and Revolution, Princeton, Princeton University Press, 1995, p. 105-106.

8. Hollis Clayson, « La Place de la Concorde in War and Peace ", dans Paris in Despair: Art and Everyday Life under Siege (1870-1871), Chicago, University of Chicago Press, 2002, p. 342. La figure de Halévy, juif, et ami très proche de Degas jusqu'à leur rupture pendant l'affaire Dreyfus, mériterait d'être reconsidérée à la lumière de cette œeuvre qui refuse l'essentialisation des identités.

9. André Dombrovski, "History, Memory, and Instantaneity in Edgar Degas's Place de la Concorde ", dans The Art Bulletin, vol. 93.2, juin 2011, p. 195-219. À l'instar de Varnedoe, l'auteur ne croit pas à l'influence de la photographie, évoquant plutôt celle de la caricature (p. 195-197).

10. Denys Sutton, "Leftspeak at Harvard ", dans Apollo, vol. $122, \mathrm{n}^{\circ} 285$, novembre 1985, p. 326-327.

11. Albert Kostenevich, Hidden Treasures Revealed: Impressionist Masterpieces and Other Important French Paintings Preserved by the State Hermitage Museum, cat. exp. (Saint-Pétersbourg, musée de l'Ermitage, 1995), Saint-Pétersbourg / New York, The Ministry of Culture of the Russian Federation and H. N. Abrams, 1995, p. 11. L'auteur avait étudié clandestinement le tableau de Degas trente ans auparavant, alors qu'il était étudiant. Il s'efforce d'expliquer comment l'Ermitage est entré en possession des œuvres et pourquoi elles sont restées invisibles pendant cinquante ans (p. 9-18). Il donne également un aperçu de la collection Gerstenberg (p. 17-18)

12. Mari Kàlman Meller, "Degas's Place de la Concorde: Vicomte Lepic and his Daughters ", dans The Burlington Magazine, $\mathrm{n}^{\circ} 145,2003$, p. 273-281. Roberta CrisciRichardson souligne que l'artiste avait inventé cette anecdote, et que René-Hilaire de Gas avait quitté Paris en 1804 , non pas en raison de ses opinions politiques, mais pour échapper à ses créanciers (Mapping Degas: Real Spaces, Symbolic Spaces and Invented Spaces in the Life and Work of Edgar Degas (1834-1917), Newcastle-upon-Tyne, Cambridge Scholars Publishing, 2015, p. 36-37.

13. Todd Porterfield, "The Obelisk at the Place de la Concorde ", dans The Allure of Empire: Art in the Service of French Imperialism in the Near East, 1798-1836, Princeton, Princeton University Press, 1998, p. 13-42.

14. Stanislas Bellanger et al., Les Étrangers à Paris, Paris, C. Warée, 1844, p. 478. La comtesse désabusée savait peut-être qu'à bien des égards le décor de la place de la Concorde avait eu un précédent dans le gigantesque service de table égyptien produit en 1804-1812 par la manufacture 
de Sèvres et orné d'un kaléidoscope de pièces architecturales pharaoniques colossales reproduites en miniature (1804-1812) ; voir T. Porterfield, " Egyptomania ", dans Art in America, vol. 82, $\mathrm{n}^{\circ} 11$, novembre 1994, p. 84-88, 149 ; Porterfield, 1998, cité n. 13, p. 140-141.

15. Thomas von Joest, Claudine de Vaulchier (dir.), Hittorff: un architecte $d u X I X^{e}$ siècle, cat. exp. (Paris, musée Carnavalet, 1986-1987), Paris, musée Carnavalet, 1986, p. 108-109.

16. L'Illustration, 12 août 1854 ; cité dans De la place Louis XV à la place de la Concorde, cat. exp. (Paris, musée Carnavalet, 1982), Paris, musée Carnavalet, 1982, p. 132.

17. Shelley Rice, Parisian Views, Cambridge, MIT Press, 1997, p. 45. Linda Nochlin rattache ces effets spéciaux à la " Platzangst ", l'agoraphobie moderne qui touche tout particulièrement les hommes de la bourgeoisie ( " Le portrait impressionniste et la construction de l'identité moderne ", dans Colin B. Bailey et al., Les Portraits de Renoir : Impression d'une époque, Paris, Gallimard, 2007).

18. Caroline Igra, « Monuments to Prior Glory: The Foreign Perspective on Post-Commune Paris ", dans Zeitschrift für Kunstgeschichte, vol. 62, n 4, 1991, p. 512-526. Voir également Ann Dumas, Degas and the Italians in Paris, Édimbourg, National Galleries of Scotland, 2003.

19. Dans une lettre datée du 21 mai 1871 adressée à Léontine De Nittis, épouse de l'artiste, Degas saluait « l'attitude qu[e Nittis] a prise d'inventer dans ce monde des peintres qui font des rues de Paris en général " (citée dans Mary Pittaluga et Enrico Piceni, De Nittis, Milan, Bramante, 1963, p. 368).

20. Jean Sutherland Boggs, Portraits by Degas, Berkeley, University of California Press, 1962, p. 93, note 16. C. Igra évoquant en détail la réception de la Place des Pyramides, signale la controverse déclenchée par la présence de l'échafaudage, jugé soit de trop, soit admirablement réaliste ( "Imagery of Destruction and Reconstruction: Giuseppe de Nittis's Forthright Approach to Post-Commune Paris ", dans Konsthistorisk Tidskrift/Journal of Art History, vol. 67, n 3, 1998, p. 163-166).

21. Linda Nochlin, "A House is not a Home: Degas and the Subversion of the Family ", dans Richard Kendall et Griselda Pollock (dir.), Dealing with Degas: Representations of Women and the Politics of Vision, Londres, HarperCollins, 1991, p. 49 (l'auteur cite ici un texte de Henri Baudrillart, La Famille et l'éducation en France, publié en 1874). Nochlin analyse la "structure formelle d'indifférence " et la " pratique générale et quasi systématique de fragmentation et le mouvement centrifuge qui caractérisent la représentation de groupes familiaux chez Degas ", notamment dans La Famille Bellelli et dans la Place de la Concorde (p. 48).

22. Harvey Buchanan, "Edgar Degas and Ludovic Lepic: An Impressionist Friendship ", dans Cleveland Studies in the History of Art, vol. 2, 1997, p. 87. Thierry Zimmer émet quelques doutes sur la date de la dissolution du mariage voir "Ludovic Napoléon Lepic, 1839-1889 ", thèse de doctorat, université Paris-Sorbonne, 1996, p. 113-115.

23. Les Amants légitimes, une pièce de 1893 dédicacée à la belle-sœur de Lepic, l'écrivaine Louise Claire Janvier Lepic, dénonce la rigidité de l'institution du mariage et le conservatisme de la nouvelle législation sur le divorce ; voir Nicholas White, « The Name of the Divorcée: Janvier and Ballot's Theatrical Critique, Mon nom ! (1892) ", dan Romance Quarterly, vol. 49, nº 3, été 2002, p. 215-227 ; idem, " Green Eyes and Purple Prose: Late Nineteenth-Century French Divorce Literature - a Proposal for Research ", dans
Rachel Langford (dir.), Depicting Desire: Gender, Sexuality, and the Family in Nineteenth-Century Europe: Literary and Artistic Perspectives, actes du colloque (Cardiff University, 2001). Oxford / Berne / Berlin, Peter Lang, 2005, p. 217. Sur le milieu politique et intellectuel fréquenté par la belle-sœur de Lepic et sa mère, ainsi que sur leurs ouvrages (publiés sous les pseudonymes Adèle Gennevraye, Genevraye ou Genevray), voir Claude Schopp, « Les dédicataires d'Alkan. I. Adèle Janvier ", dans Bulletin de la Société Alkan, $\mathrm{n}^{\text {os }}$ 71-73, juillet 2007 - mars 2008, p. 25-30.

24. Clayson, 2002, cité n. 8, p. 331-335. Voir également De la place Louis XV..., 1982, cité n. 16, p. 136.

25. Léon de Lora (pseudonyme d'Alexandre Pothey), "Lepic ", dans Galerie contemporaine, littéraire et artistique, 2, no 15,1877 , s.p.

26. Buchanan, 1997, cité n. 22, p. 32-120.

27. Zimmer, 1996, cité n. 22, p. 75.

28. Buchanan, 1997, cité n. 22, p. 68. Selon l'auteur, le chien du tableau est fait sûrement partie de la progéniture d'Abreckt (p. 81-83).

\section{Buchanan, 1997, cité n. 22, p. 68, 70.}

30. Ruth Clifford Engs, The Eugenics Movement: An Encyclopedia, Westport, Greenwood Press, 2005, p. xiii-xiv, 82.

31. Harriet Ritvo, The Animal Estate: The English and Other Creatures in the Victorian Age, Cambridge, Harvard University Press, 1987, p. 84. Lepic offrit des textes et des illustrations, ainsi que des facsimilés d'outils préhistoriques au musée des Antiquités nationales de Saint-Germain-en-Laye. En 1869, il devint membre de la Société d'anthropologie. Sous la III ${ }^{e}$ République, il dirigea le musée de la Préhistoire d'Aix-les-Bains (autrefois appelé " musée Lepic » et aujourd'hui musée d'Archéologie nationale), de sa fondation en 1872, jusqu'à sa mort (voir. Buchanan, 1997, cité n. 22, p. 39-45). Voir également Thierry Zimmer, Ludovic-Napoléon Lepic, 1839-1889, "Le patron ", cat. exp. (Berck-sur-Mer, musée d'Opale Sud, 2013), Berck-sur-Mer, musée d'Opale Sud, 2013, p. 70-77 et Hélène Chew, Ludovic Napoléon Lepic, peintre et archéologue sous Napoléon III : regards sur les collections du musée d'Archéologie nationale, Saint-Germain-en-Laye, musée d'Archéologie nationale, 2017.

32. Dans l'album de l'exposition canine de 1863, la photographie montre le chien de Lepic de profil, ce qui est inhabituel ; la plupart des portraits sont plus informels et montrent l'animal de trois-quarts (voir Exposition canine $d u$ Bois de Boulogne Collection du Journal des chasseurs, Bureau du Journal, 1863). Voir également, à titre de comparaison, l'Exposition de 1884 (Société centrale pour l'amélioration des races de chiens en France, 1884) et Léon Crémière (dir.), Le Chien illustré. Types de races canines, avec notices, Paris, Firmin Didot, 1884. Les articles du catalogue indiquent le pedigree, l'âge, la parenté et les dimensions de l'animal, ainsi que le nom de son propriétaire.

33. Société du Jardin zoologique d'acclimatation du Bois de Boulogne, Exposition universelle des races canines de 1865, Paris, P. Dupont, 1865, p. 60. L'exposition de 1865 occupait le Cours-la-Reine et la partie ouest de la place de la Concorde.

34. Cité dans Richard Thomson, «"Les Quat' pattes": the Image of the Dog in Late Nineteenth-Century French Art ", dans Art History, vol. 5, n 3, septembre 1982, p. 324-325.

35. Ségolène Le Men, Courbet, Paris, Citadelles et Mazenot, 2007, p. 322-323. 
36. Antoine Dorfeuille, Lettre d'un chien aristocrate à son maître, aristocrate aussi et fugitif de Toulouse, Toulouse, Chez Viallannes, s.d. [1793], p. 7-8

37. Anne Middleton Wagner, Jean-Baptiste Carpeaux: Sculptor of the Second Empire, New Haven / Londres, Yale University Press, 1986, p. 193-194.

38. Edgar Degas, Lettres de Degas, Marcel Guérin (éd.), Paris, Grasset, 1945, p. 149-151 ; cité également dans Buchanan, 1997, cité n. 22, p. 69-70.

39. Zimmer, 1996, cité n. 22, p. 391-392, et Buchanan, 1997 cité n. 22, p. 36-39. Ces gravures figureront également dans la première Exposition impressionniste, en 1874 .

40. La toile de Degas présente de nombreuses similitudes avec certains détails de la fresque : concentration des protagonistes au premier plan du champ pictural, se détachant sur le fond architectural ; le chien placé dans le coin inférieur gauche ; la parabole décrite par les éléments du paysage qui se déploient derrière le cou du chien. On note aussi les deux pendus accrochés à un gibet, à l'aplomb de la tête du chien de Pisanello ; les deux sites en plein air sont donc des lieux d'exécution, une coïncidence qui ne manque pas de sel.

41. Francis Ames-Lewis et Joanne Wright, Drawings of the Italian Renaissance Workshop, cat. exp. (Nottingham, University Art Gallery, 1983), Londres, Victoria \& Albert Museum, 1983, p. 97. Des copies du Codex Vallardi figurent dans le cahier 13 de Degas (BnF Dc327d réserve, p. 49), que l'artiste a utilisé à Paris, à Florence et à Gênes entre 1858 et 1860 . On trouve à la page 49 le " croquis de la croupe d'un cheval orienté à gauche. En-dessous, une copie du dessin de Pisanello, la Tête d'aigle "; voir Theodore Reff, The Notebooks of Edgar Degas: A Catalogue of the Thirty-Eight Notebooks in the Bibliothèque Nationale and Other Collections, Oxford, Clarendon Press, 1976, vol. 1, p. 78,80 .

42. Sur la première attribution, voir Giuseppe Vallardi, Disegni di Leonardo da Vinci possedati da Giuseppe Vallardi, Milan, Pietro Agnelli, 1855 ; sur la seconde, voir R. Reiset, "Une visite aux musées de Londres en 1876 " (deuxième article), dans Gazette des beaux-arts, vol. 15, n² 2, p. 119-122. $C^{\prime}$ est sans doute dans la seconde moitié du Xve siècle que les héritiers du fonds d'œuvres de l'atelier de Pisanello collèrent les dessins dans l'album connu sous le nom de Codex Vallardi ; voir Albert J. Elen, Italian Late-Medieval and Renaissance Drawing-Books from Gionannino de' Grassi to Palma Giovane. A Codicological Approach [1955], Leyde, Proefschrift, 1995, p. 130-135.

43. Lettre d'Auguste de Gas adressée à son fils à Florence, datée de Paris, 25 novembre 1858 ; Henry Loyrette, Degas, Paris, Fayard, 1991, p. 48.

44. Lettre d'Auguste de Gas adressée à son fils à Florence, datée de Paris, 4 janvier 1859 ; Loyrette, 1991, cité n. 43, p. 48. Durant ces années, Degas opère une fusion évidente entre affiliations artistiques et familiales, comme on peut le voir notamment dans La Famille Bellelli; voir Louis Alexander Waldman, "Degas, Berenson and Italy ", dans Rosa Spinillo (dir.), I Bellelli e Degas. Iconografia e storia di una famiglia italiana, Rome, Palombi, 2014, p. 12-17. Les livres de modèles représentent les objets comme "sous vide, avec un raffinement qui les écarte du réel " (Ames-Lewis et Wright, 1983, cité n. 41, p. 98).

45. Robert W. Scheller, Exemplum. Model-Book Drawings and the Practice of Artistic Transmission in the Middle Ages (ca. 900
- ca. 1470), Michael Hoyle (Eng. transl.), Amsterdam University Press, 1995, p. 6, 7, 13.

46. Elen, 1995, cité n. 42, p. 205. Voir également Todd Porterfield, "Transfer, Repetition, and Reversal in Degas's Late Drawings ", manuscrit inédit, Midwest Art History Conference, 1983.

47. Robert Rey, La Renaissance du sentiment classique dans la peinture française à la fin du XIXe siècle. Degas, Renoir, Gauguin, Cézanne, Seurat, Paris, Éditions G. Van Oest, Les Beaux-arts, 1931, p. 135-137, 39-42.

48. Voir Éric Michaud, Histoire de l'art. Une discipline à ses frontières, Paris, Hazan, 2005 ; idem, Les Invasions barbares une généalogie de l'histoire de l'art, Paris, Gallimard, 2015.

49. Le père de Lepic mourut en avril 1875, l'année où le tableau fut peint et, comme en fit largement état Le Gaulois à l'automne, le château familial d'Andrésy fut cambriolé, vandalisé, les rideaux déchirés, les meubles détruits, les papiers déchirés et éparpillés ("Informations générales", dans Le Gaulois, vol. VIII, n 2550, 11 octobre 1875).

50. Robert L. Herbert, qui date ce dessin de 1882-1883, pense qu'il s'agit d'une vue de l'esplanade de l'Orangerie (R. L. Herbert [dir.], Georges Seurat, 1859-1891, cat. exp. [Paris, Galeries nationales du Grand Palais / New York, The Metropolitan Museum of Art, 1991-1992], New York, The Metropolitan Museum of Art, 1991, p. 89-90, 92). Pour Jonathan Crary, le dessin, qui fait partie des " images d'isolement et de séparation " de Seurat montre, de façon cauchemardesque, que les rêves collectifs " ne peuvent même plus être rappelés " (Suspensions of Perception: Attention, Spectacle, and Modern Culture, Cambridge, MIT Press, 1999, p. 184-186). Quant à Werner Hofmann, il déclare, à propos de la toile de Degas : " Mais l'isolement ne survient réellement que lorsqu'une impulsion centrifuge naît d'éléments statiques reliés entre eux, et ouvre la voie à créer des forces centrifuges qui vident l'espace et déstabilisent les individus qui l'occupent " (Hofmann, 2007, cité n. 5, p. 105).

51. Georges Bataille, "Architecture", dans Documents, vol. 1, n 2, 1929, p. 117 ; "Musée ", dans Documents, vol. 2, n 5, 1930, p. 300 ; "L'obélisque ", dans Mesures, 15 avril 1930, p. 35-50. Voir la belle analyse de Denis Hollier dans La Prise de la Concorde : essais sur Georges Bataille, Paris, Gallimard, 1974. L'édition américaine du livre comporte une introduction qui n'a pas été reprise dans la deuxième édition française (La Prise de la Concorde : suivi de Les dimanches de la vie : essais sur Georges Bataille, Paris, Gallimard, 1993).

52. Bataille, 1929, cité n. 51, p. 117. 
actualité en histoire de l'art 